\section{Cystin im Urin}

W. G. Guder

München, Deutschland

Synonym(e) Aminosäure (Cystin (Cys-Cys), Arginin, Lysin, Ornithin)-Transportstörung; Cystinkristalle im Sediment

Englischer Begriff cystinuria; cystin stones; cystin cristals in urinary sediment

Definition Kristallbildung von Cystin bei vermehrter Ausscheidung dieser Aminosäure im Urin auf der Basis der autosomal rezessiv vererbbaren Cystinurie, die durch einen Defekt des Aminosäurecarriers für Cystin, > Ornithin und - Lysin im proximalen Tubulus beruht.

Struktur Dimer von $\alpha$-Amino- $\beta$-Mercaptopropionsäure (Cystein); \ Aminosäuren.

Molmasse $242 \mathrm{~g}$.

Pathophysiologie Aufgrund einer Mutation des Gens für den Transporter von dibasischen Aminosäuren (Arginin, Lysin und Ornithin) kommt es zu einer Hemmung der Resorption des im Tubuluslumen durch Oxidation aus Cystein entstehenden Cystins. Dies wird nach Harnkonzentrierung im Sammelrohr bei physiologischem Harn-pH (sauer) unlöslich, bildet Cystinkristalle im Harnsediment und in der Folge größere Harnkonkremente. Wenn die Größe der Kristalle die Weite des ableitenden Harnwegs überschreitet, kommt es zur klinischen Symptomatik der Urolithiasis.

Untersuchungsmaterial Zur Untersuchung auf Kristalle im Harnsediment genügt eine Portion $\$ Spontanurin. Wegen der geringen Löslichkeit des Cystins ist eine Quantifizierung der
Cystinausscheidung nur nach Auflösung aller Kristalle aus 24-Stunden-Sammelurin möglich. Jedes Konkrement sollte auf Cystin als Bestandteil untersucht werden.

Probenstabilität 24 Stunden gekühlt, wenn Cystein und Cystin gemeinsam bestimmt werden; $\mathrm{pH}>7,5$ verhindert Kristallisation.

Analytik Qualitativer Nachweis der Kristalle im Harnsediment, Steinanalyse mit $>$ Infrarot-Spektrometrie oder Röntgendiffraktion. Quantitative Bestimmung mit HPLC ( $\triangleright$ Chromatographie) oder am Aminosäureanalysator.

Internationale Einheit $\mathrm{mmol} / 24 \mathrm{~h}$ oder $\mathrm{mg} / 24 \mathrm{~h}$.

Umrechnungsfaktor $\mathrm{mmol} \times 235=\mathrm{mg} ; \mathrm{mg} \times 0,00425=$ mmol.

Referenzbereich Frauen, Männer, Kinder ab dem 12 Lebensjahr: $0,17-0,33 \mathrm{mmol} / 24 \mathrm{~h}(40-80 \mathrm{mg} / 24 \mathrm{~h})$.

Indikation Jeder abgehende Harnstein sollte untersucht werden. Bei Steinsymptomatik Sedimentuntersuchung auf Cystinkristalle. Darüber hinaus achtet man auf Cystinkristalle bei jeder Harnsedimentuntersuchung. Bei familiärer Belastung und vor Therapie eines nachgewiesenen Cystinsteins quantitative Cystinbestimmung.

Diagnostische Wertigkeit Der Nachweis eines Cystinsteins oder wiederholter Cystinkristallbildung im Harn ist für das Vorliegen einer Cystinurie beweisend.

\section{Literatur}

Hesse A, Jahnen A, Klocke K, Nolde A, Scharrel O (1994) Nachsorge bei Harnsteinpatienten. Gustav Fischer Verlag, Jena 